\title{
A new arrival: evidence about differential diagnosis
}

You are a primary care physician seeing a 53 year old man who was examined 2 days ago in an emergency department for an episode of syncope. He had been waiting in a long queue when he felt lightheaded and nauseated; then he lost consciousness, with no witnessed seizure activity. He has been healthy, with no known cardiac or neurological disease. In the emergency department, his vital signs and findings on cardiac and neurological examinations had been normal, as were his blood count, blood glucose concentration, and 12 lead electrocardiogram. He was given no diagnosis, and he is now worried about what caused this episode and whether it will recur. Of the many causes of syncope, you wonder which should be sought in this patient.

"Differential diagnosis" refers to the methods by which we consider the possible causes of patients' clinical findings before making final diagnoses. ${ }^{12}$ At its heart, differential diagnosis involves acts of selection: we consider a patient's illness and choose which disorders to pursue further. Is there high quality research that could guide our selections, such as evidence about the frequency of disorders that can cause clinical problems like syncope? If so, wouldn't it be grand if someone would find it, summarise it, and tell us about it?

Beginning with this issue, Evidence-Based Medicine will do just that-search for the best new evidence about differential diagnosis as it is published, put it through the same rigorous process used for other types of evidence, ${ }^{3}$ and publish a structured abstract with a clinical commentary. To be selected for inclusion under the new heading "differential diagnosis," original articles must pass all the following quality criteria:

- The study must report a cohort (consecutive or random sample) of patients presenting with a similar, initially undiagnosed but well defined clinical problem

- The clinical setting must be well described

- The diagnostic evaluation must be both well described and credible

- For $\geqslant 80 \%$ of those patients evaluated, either the diagnoses must be established (by appropriate investigation), or to minimise missed diagnoses, there must be a follow up of $\geqslant 1$ month for acute disorders and $\geqslant 1$ year for chronic or recurring disorders.

Beyond these fundamentals, preference will be given to studies that use stronger methods to reduce bias, including prospective designs, explicit diagnostic criteria, and standardised evaluations applied to sufficient numbers of patients. Any clinical problem could qualify, but preference will be given to studies of problems that in the editors' judgment are most relevant for the targeted readers of our journal, especially those in primary care.

In this issue, the abstracted article about syncope by Ammirati et al illustrates these criteria well. ${ }^{4}$ Syncope is common in practice, with numerous causes ( 1 text lists $>50),{ }^{5}$ ranging from simple fainting to life threatening arrhythmias.

The range of diagnostic strategies is also great, as may be our uncertainty about which tests would be best. Although a standard text may list the diagnostic possibilities, high quality evidence about differential diagnosis can tell us about the probabilities of the disorders that cause syncope.
The main results of research about differential diagnosis can be expressed as the number and percentages of patients who are found to have each underlying disorder. For instance, in the study by Ammirati et al, of 195 patients presenting with syncope to any of 9 Italian community hospitals, we find that 69 (35\%) were diagnosed with neurovascular reflex disorders. From this 35\% frequency in the whole sample, we can estimate the disease probability of neurovascular reflex disorders for any 1 patient from the sample to be 0.35 or $35 \%$. In general, conditions diagnosed frequently will have relatively high disease probabilities in the study, whereas those seldom diagnosed will have low disease probabilities.

We can use the study's disease probabilities as starting points for estimating pretest probability in our own patients and then adjust these probabilities, taking into consideration features of our patients or our practices. ${ }^{6}$ For instance, the study by Ammirati et al reports disease probabilities of $35 \%$ for neurovascular reflex disorders and 21\% for cardiac disorders. The scenario patient's history (prolonged standing, then premonitory symptoms, then syncope) suggests a neurovascular reflex cause, which raises the pretest probability of a neurovascular reflex disorder for this patient. The absence of cardiac findings on the history, examination, and electrocardiogram lowers the probability of cardiac disorders for this patient.

Once we have them, pretest probabilities can help with 3 diagnostic decisions. Firstly, pretest probabilities can help us to decide whether to pursue specific disorders as explanations for our patient's illness. A sensible strategy is to choose the most probable disorder as the "working diagnosis" we plan to confirm and then to select from the remaining disorders those few that are likely, serious, and treatable enough to be the "active alternatives" that we plan to exclude. ${ }^{7}$ These selections (working diagnosis and active alternatives) form the short list of our initial differential diagnosis, and we would pursue these disorders first. If the short list does not lead us to a diagnosis, we could then consider other disorders with lower probabilities. Secondly, we can use pretest probabilities when choosing which diagnostic tests to use or when interpreting their results. ${ }^{8}$ Thirdly, we can also use them when deciding whether to forgo any testing and proceed with treatment. ${ }^{78}$

But why should we bother using evidence for differential diagnosis? Why not rely solely on our clinical experience when estimating pretest probability? Our case memories are as close as our thoughts and are directly calibrated to our practices. But using remembered cases requires us to have had sufficient experience with this problem (dozens or hundreds of cases) and for us to be able to accurately recall this experience. ${ }^{6}$ Several biases can distort the frequencies we estimate from remembered cases, ${ }^{9}{ }^{10}$ as we tend to recall striking or recent individual numerator cases without the proper denominators.

We can't and shouldn't stop using remembered cases to estimate pretest probability. Still, it makes sense to know when our memories may be prone to ignorance or bias. For instance, when we've had little or no previous experience with a problem or when a newly described clinical problem is reported, we need external evidence to educate our estimates of pretest probability. Alternatively, when we've 
recently changed practice settings or when we've recently diagnosed an unusual cause for a clinical problem, external evidence can help us to recalibrate our estimates of pretest probability. In addition, when important new research about differential diagnosis is published, we need to examine it to decide whether to recalibrate our established estimates of pretest probability.

With the addition of "differential diagnosis" to its family of evidence, Evidence-Based Medicine aims to serve all of these needs, particularly the last-keeping up with new research. We hope this new arrival will help you to become better and faster at estimating pretest probabilities and selecting differential diagnoses.

\section{W SCOTT RICHARDSON, MD University of Texas Health Sciences Center San Antonio, Texas, USA \\ PAUL GLASZIOU, MBBS, PhD University of Oueensland Herston, Queensland, Australia \\ WALTER A POLASHENSKI, MD University of Rochester Rochester, New York, USA \\ MARK C WILSON, MD, MPH Wake Forest University Winston-Salem, North Carolina, USA}

1 Sox HC, Blatt MA, Higgins MC, et al. Medical decision making. Boston: Butterworth, 1988.

2 Barondess JA, Carpenter CC, editors. Differential diagnosis. Philadelphia: Lea \& Febiger, 1994.

3 Sackett DL, Haynes RB. 13 steps, 100 people, and 1000000 thanks [editorial]. ACP J Club 1997 Jul-Aug;127:A14.

4 A simple algorithm improved diagnostic performance in patients presenting with syncope [abstract]. Evidence-Based Medicine 2000 Nov-Dec;5:191. Abstract of Ammirati F, Colivicchi F, Santini M, on behalf of the investigators of the OESIL study. Diagnosing syncope in clinical practice. Implementation of a simplified diagnostic algorithm in a multicentre prospective trial-the OESIL 2 Study (Osservatorio Epidemiologico della Sincope nel Lazio). Eur Heart J 2000;21:935-40.

5 Adler SN, Gasbarra DB, Adler-Klein D. A pocket manual of differential diagnosis. 4th edition. Philadelphia: Lippincott, Williams \& Wilkins; 2000.

6 Richardson WS. Where do pretest probabilities come from? [EBM note]. Evidence-Based Medicine 1999 May-Jun;4:68-9.

7 Richardson WS, Wilson MC, Guyatt GH, et al, for the Evidence-Based Medicine Working Group. Users' guides to the medical literature. XV. How to use an article about disease probability for differential diagnosis. JAMA 1999;281:1214-9.

8 Sackett DL, Straus SE, Richardson WS, et al, editors. Evidence-based medicine: how to practice and teach EBM. 2nd edition. Edinburgh: Churchill Livingstone, 2000

9 Tversky A, Kahneman D. Judgment under uncertainty: heuristics and biases. Science 1974;185:1124-31.

10 Dawson NV, Arkes HR. Systematic errors in medical decision-making: judgment limitations.J Gen Intern Med 1987;2:183-7.

\section{Journals reviewed for this issue*}

Acta Obstet Gynecol Scand
Age Ageing
Am J Cardiol
Am J Med
Am J Obstet Gynecol
Am J Psychiatry
Am J Public Health
Am J Respir Crit Care Med
Am J Surg
Ann Emerg Med
Ann Intern Med
Ann Med
Ann Surg
Arch Dis Child
Arch Fam Med
Arch Gen Psychiatry
Arch Intern Med

Acta Obstet Gynecol Scand

Am J Med

Am J Respir Crit Care Med

Ann Intern Med

Arch Intern Med
Diabetes Care

Fertil Steril

Gastroenterology

Gut

Heart

Hypertension

JAMA

J Am Board Fam Pract

J Am Coll Cardiol

J Am Coll Surg

J Am Geriatr Soc

J Clin Epidemiol

$\mathrm{J}$ Fam Pract

J Gen Intern Med

$\mathrm{J}$ Infect Dis

J Intern Med
J Neurol Neurosurg Psychiatry

J Pediatr

J Vasc Surg

Lancet

Med Care

Med J Aust

$\mathrm{N}$ Engl J Med

Neurology

Obstet Gynecol

Pain

Pediatrics

Rheumatology

Spine

Stroke

Surgery

Thorax

*Approximately 60 additional journals are reviewed. This list is available on request.

How to cite material from Evidence-Based Medicine

Citation of material from the Notebook

Milne R, Hicks N. Evidence-based purchasing [EBM Note]. Evidence-Based Medicine 1996 May-Jun;1:101-2.

Citation for material taken from a structured abstract, written without attribution by a staff member

Antihypertensive drugs decrease mortality, coronary events, and stroke in elderly persons [abstract]. Evidence-Based Medicine 1996 May-Jun;4:105. Abstract of: Pearce KA, Furberg CD, Rushing J. Does antihypertensive treatment of the elderly prevent cardiovascular events or prolong life? A meta-analysis of hypertension treatment trials. Arch Fam Med 1995;4:943-50.

Citation for material taken from a commentary to an article

Olds D. Commentary on "Home visiting programmes reduce childhood injury." Evidence-Based Medicine 1996 May-Jun;4:112. Comment on: Roberts I, Kramer MS, Suissa S. Does home visiting prevent childhood injury? A systematic review of randomised controlled trials. BMJ 1996;312:29-33. 